\title{
Automorphisms of a Polynomial Ring Which Admit Reductions of Type I
}

\author{
By
}

Shigeru KURODA*

\begin{abstract}
Recently, Shestakov-Umirbaev solved Nagata's conjecture on an automorphism of a polynomial ring. To solve the conjecture, they defined notions called reductions of types I-IV for automorphisms of a polynomial ring. An automorphism admitting a reduction of type I was first found by Shestakov-Umirbaev. Using a computer, van den Essen-Makar-Limanov-Willems gave a family of such automorphisms. In this paper, we present a new construction of such automorphisms using locally nilpotent derivations. As a consequence, we discover that there exists an automorphism admitting a reduction of type I which satisfies some degree condition for each possible value.
\end{abstract}

\section{§1. Introduction}

Let $k$ be a field of characteristic zero, and $k[\mathbf{x}]=k\left[x_{1}, \ldots, x_{n}\right]$ the polynomial ring in $n$ variables over $k$. We will identify an endomorphism $F \in$ End $_{k} k[\mathbf{x}]$ with the $n$-tuple $\left(f_{1}, \ldots, f_{n}\right)$ of elements of $k[\mathbf{x}]$, where $f_{i}=F\left(x_{i}\right)$ for each $i$. Then, $F$ is invertible if and only if $k\left[f_{1}, \ldots, f_{n}\right]=k[\mathbf{x}]$. If this is the case, the $\operatorname{sum} \operatorname{deg} F:=\sum_{i=1}^{n} \operatorname{deg} f_{i}$ of the total degrees of $f_{i}$ 's is necessarily at least $n$. An automorphism $F \in$ Aut $_{k} k[\mathbf{x}]$ is said to be affine if $\operatorname{deg} F=n$, and elementary if there exist $i \in\{1, \ldots, n\}$ and a polynomial $\phi \in k[\mathbf{x}]$ not depending on $x_{i}$ such that $f_{i}=x_{i}+\phi$ and $f_{j}=x_{j}$ for each $j \neq i$. We say that $F$ admits an elementary reduction if there exists an elementary automorphism $G$ such that $\operatorname{deg}(F \circ G)<\operatorname{deg} F$. Note that $F$ admits an elementary

Communicated by S. Mori. Received August 12, 2008.

2000 Mathematics Subject Classification(s): Primary 14R10; Secondary 13N15.

Partly supported by the Grant-in-Aid for Young Scientists (Start-up) 19840041, The

Ministry of Education, Culture, Sports, Science and Technology, Japan.

* Department of Mathematics and Information Sciences, Tokyo Metropolitan University, 1-1 Minami-Osawa, Hachioji, Tokyo 192-0397, Japan. 
reduction if and only if there exists $\phi \in k\left[f_{1}, \ldots, f_{i-1}, f_{i+1}, \ldots, f_{n}\right]$ such that $\operatorname{deg}\left(f_{i}+\phi\right)<\operatorname{deg} f_{i}$ for some $i$. The subgroup $\mathrm{T}_{k} k[\mathbf{x}]$ of the automorphism group Aut $_{k} k[\mathbf{x}]$ generated by affine automorphisms and elementary automorphisms is called the tame subgroup, and each element of $\mathrm{T}_{k} k[\mathbf{x}]$ is called a tame automorphism.

By Jung [3], it follows that $\mathrm{T}_{k} k[\mathbf{x}]=\operatorname{Aut}_{k} k[\mathbf{x}]$ when $n=2$. In fact, he showed that each $F \in$ Aut $_{k} k[\mathbf{x}]$ for $n=2$ admits an elementary reduction whenever $\operatorname{deg} F>2$. Thereby,

$$
\operatorname{deg} F>\operatorname{deg}\left(F \circ G_{1}\right)>\cdots>\operatorname{deg}\left(F \circ G_{1} \circ \cdots \circ G_{r}\right)=2
$$

for some elementary automorphisms $G_{1}, \ldots, G_{r}$ of $k[\mathbf{x}]$. Note that a similar result for a field $k$ of an arbitrary characteristic was given by van der Kulk [4].

Now, assume that $n=3$. Nagata [8] conjectured that the automorphism

$$
\left(x_{1}-2\left(x_{1} x_{3}+x_{2}^{2}\right) x_{2}-\left(x_{1} x_{3}+x_{2}^{2}\right)^{2} x_{3}, x_{2}+\left(x_{1} x_{3}+x_{2}^{2}\right) x_{3}, x_{3}\right)
$$

of $k[\mathbf{x}]$ is not tame. In 2003, this well-known conjecture was finally solved in the affirmative by Shestakov-Umirbaev [9], [10]. They defined four types of reductions, said to be of types I, II, III and IV, for elements of Aut $k$ k[x]. Then, they showed that each non-affine element of $\mathrm{T}_{k} k[\mathbf{x}]$ admits an elementary reduction or one of these four types of reductions. One can easily check that Nagata's automorphism admits none of these reductions. Therefore, Nagata's conjecture holds true.

In the present paper, we study automorphisms admitting reductions of type I.

Definition 1.1 [10, Definition 1]. Assume that $n=3$. We say that an automorphism $F=\left(f_{1}, f_{2}, f_{3}\right)$ of $k[\mathbf{x}]$ admits a reduction of type $I$ if the following conditions hold:

(i) There exists an odd number $s \geq 3$ such that $\operatorname{deg} f_{1}: \operatorname{deg} f_{2}=2: s$.

(ii) $\operatorname{deg} f_{1}<\operatorname{deg} f_{3} \leq \operatorname{deg} f_{2}$.

(iii) $\bar{f}_{3}$ does not belong to $k\left[\bar{f}_{1}, \bar{f}_{2}\right]$, where $\bar{f}$ denotes the highest homogeneous part of $f$ for each $f \in k[\mathbf{x}]$.

(iv) There exist $\alpha \in k \backslash\{0\}$ and $\phi \in k\left[f_{1}, f_{2}-\alpha f_{3}\right]$ such that $\operatorname{deg}\left(f_{3}+\phi\right)<$ $\operatorname{deg} f_{3}$ and $\operatorname{deg}\left[f_{1}, f_{3}+\phi\right]<\operatorname{deg} f_{2}+\operatorname{deg}\left[f_{1}, f_{2}-\alpha f_{3}\right]$. Here, we define

$$
\operatorname{deg}[f, g]=\max \left\{\operatorname{deg}\left(\frac{\partial f}{\partial x_{i}} \frac{\partial g}{\partial x_{j}}-\frac{\partial f}{\partial x_{j}} \frac{\partial g}{\partial x_{i}}\right) \mid 1 \leq i<j \leq 3\right\}+2
$$

for each $f, g \in k[\mathbf{x}]$.

We also say that $F$ admits a reduction of type I if $\left(f_{\sigma(1)}, f_{\sigma(2)}, f_{\sigma(3)}\right)$ satisfies (i)-(iv) for some permutation $\sigma$ of $\{1,2,3\}$. 
We mention that the conditions listed above imply that $\operatorname{deg}\left(f_{2}-\alpha f_{3}\right)=$ $\operatorname{deg} f_{2}$ and that $f_{1}$ and $f_{2}-\alpha f_{3}$ form a "*-reduced pair", which are also included in the definition of reduction of type I by Shestakov-Umirbaev.

Note that $\left(f_{1}, f_{2}-\alpha f_{3}, f_{3}\right)$ admits an elementary reduction by (iv), while $\left(f_{1}, f_{2}, f_{3}\right)$ does not (cf. [10, Proposition 1]). Shestakov-Umirbaev [10, Example 1] gave the first example of a tame automorphism which admits a reduction of type I in case of $s=3$. Van den Essen-Makar-Limanov-Willems [1] constructed a family of such automorphisms when $s=3,5,7$ using a computer. Reductions of types II, III and IV are also defined theoretically [10], but no automorphisms admitting these reductions are found. To study the structures of $\mathrm{Aut}_{k} k[\mathbf{x}]$ and $\mathrm{T}_{k} k[\mathbf{x}]$, it is of great importance to investigate automorphisms admitting reductions of these four types.

The purpose of this paper is to construct new automorphisms of $k[\mathbf{x}]$ which admit reductions of type I by employing the theory of locally nilpotent derivations. As a consequence, we discover that there exists a tame automorphism admitting a reduction of type I such that $\operatorname{deg} f_{1}: \operatorname{deg} f_{2}=2: s$ for each odd number $s \geq 3$.

The author would like to thank Prof. Hiraku Kawanoue for informing him of an example of polynomials discussed in Section 4, to Prof. Eric Edo for many invaluable comments, and to Prof. Gene Freudenburg for helpful discussions.

\section{§2. Tame Automorphisms Admitting Reductions of Type I}

Before stating our main result, we prove a lemma. In what follows, we assume that $n=3$.

Lemma 2.1. Let $s \geq 3$ be an odd number, and $H=\left(h_{1}, h_{2}, h_{3}\right)$ a tame automorphism of $k[\mathbf{x}]$ such that

$$
\operatorname{deg} h_{1}: \operatorname{deg} h_{2}: \operatorname{deg} h_{3}=2: s: 1, \quad \frac{s-1}{2} \operatorname{deg} h_{1}<\operatorname{deg}\left(c h_{1}^{s}+h_{2}^{2}\right)<\operatorname{deg} h_{2}
$$

for some $c \in k \backslash\{0\}$. Then, $H^{\prime}=\left(h_{1}, h_{2}^{\prime}, h_{3}^{\prime}\right)$ is a tame automorphism admitting a reduction of type I for which $\operatorname{deg} h_{2}^{\prime}=\operatorname{deg} h_{2}$ and $\operatorname{deg} h_{3}^{\prime}=\operatorname{deg}\left(c h_{1}^{s}+h_{2}^{2}\right)$. Here, $h_{2}^{\prime}=h_{2}+h_{3}+c h_{1}^{s}+h_{2}^{2}$ and $h_{3}^{\prime}=h_{3}+c h_{1}^{s}+h_{2}^{2}$.

Proof. Let $G_{1}$ and $G_{2}$ be elementary automorphisms of $k[\mathbf{x}]$ defined by $G_{1}\left(x_{3}\right)=x_{3}+c x_{1}^{s}+x_{2}^{2}, G_{2}\left(x_{2}\right)=x_{2}+x_{3}$ and $G_{i}\left(x_{j}\right)=x_{j}$ for $(i, j) \neq$ $(1,3),(2,2)$. Then, $H^{\prime}=H \circ G_{1} \circ G_{2}$. Hence, $H^{\prime}$ is a tame automorphism of $k[\mathbf{x}]$, since so is $H$. By assumption, $\operatorname{deg} h_{2}$ is greater than $\operatorname{deg} h_{3}$ and $\operatorname{deg}\left(c h_{1}^{s}+h_{2}^{2}\right)$, 
while $\operatorname{deg} h_{3}$ is less than $\operatorname{deg}\left(c h_{1}^{s}+h_{2}^{2}\right)$. Hence, $\operatorname{deg} h_{2}^{\prime}=\operatorname{deg} h_{2}$ and $\operatorname{deg} h_{3}^{\prime}=$ $\operatorname{deg}\left(c h_{1}^{s}+h_{2}^{2}\right)$. It follows that

$$
l \operatorname{deg} h_{1}<\operatorname{deg} h_{3}^{\prime}<\operatorname{deg} h_{2}^{\prime}=\frac{s}{2} \operatorname{deg} h_{1}<(l+1) \operatorname{deg} h_{1}, \text { where } l=\frac{s-1}{2} .
$$

This implies that $\bar{h}_{3}^{\prime}$ does not belong to $k\left[\bar{h}_{1}, \bar{h}_{2}^{\prime}\right]$, since $\operatorname{deg} h_{3}^{\prime}<\operatorname{deg} h_{2}^{\prime}$. If $\alpha=1$, then $h_{2}^{\prime}-\alpha h_{3}^{\prime}=h_{2}$, so $\phi:=-c h_{1}^{s}-h_{2}^{2}$ is contained in $k\left[h_{1}, h_{2}^{\prime}-\alpha h_{3}^{\prime}\right]$. The total degree of $h_{3}^{\prime}+\phi=h_{3}$ is less than $\operatorname{deg} h_{3}^{\prime}$. In addition,

$$
\begin{aligned}
& \operatorname{deg}\left[h_{1}, h_{3}^{\prime}+\phi\right]=\operatorname{deg}\left[h_{1}, h_{3}\right] \leq \operatorname{deg} h_{1}+\operatorname{deg} h_{3} \leq \operatorname{deg} h_{2} \\
& \quad=\operatorname{deg}\left(h_{2}^{\prime}-\alpha h_{3}^{\prime}\right)<\operatorname{deg}\left(h_{2}^{\prime}-\alpha h_{3}^{\prime}\right)+\operatorname{deg}\left[h_{1}, h_{2}^{\prime}-\alpha h_{3}^{\prime}\right] .
\end{aligned}
$$

Therefore, $\left(h_{1}, h_{2}^{\prime}, h_{3}^{\prime}\right)$ satisfies all the conditions of Definition 1.1.

Now, let $p$ and $q$ be natural numbers, and consider triangular derivations $D$ and $E$ of $k[\mathbf{x}]$ defined by

$$
\begin{array}{cl}
D\left(x_{1}\right)=x_{2}^{q+1}, & D\left(x_{2}\right)=0, \quad D\left(x_{3}\right)=(p+1) x_{1}^{p} x_{2}^{q}, \\
E\left(x_{1}\right)=2 x_{3}, & E\left(x_{2}\right)=2(p+1) x_{1}^{p}, \quad E\left(x_{3}\right)=1 .
\end{array}
$$

Here, we say that a $k$-derivation $\Delta$ of $k[\mathbf{x}]$ is triangular if $\Delta\left(x_{\sigma(i)}\right)$ belongs to $k\left[x_{\sigma(1)}, \ldots, x_{\sigma(i-1)}\right]$ for each $i$ for some permutation $\sigma$ of $\{1, \ldots, n\}$. If this is the case, $\Delta$ is locally nilpotent, i.e., $\Delta^{l}(f)=0$ for sufficiently large $l$ for each $f \in k[\mathbf{x}]$. In particular,

$$
f_{i}=\sum_{l=0}^{\infty} \frac{D^{l}\left(x_{i}\right)}{l !}, \quad g_{i}=\sum_{l=0}^{\infty} \frac{E^{l}\left(x_{i}\right)}{l !}\left(-x_{3}\right)^{l}
$$

are elements of $k[\mathbf{x}]$ for each $i$. We set $F=\left(f_{1}, f_{2}, f_{3}\right), G=\left(g_{1}, g_{2}, x_{3}\right)$, and define $h_{1}=F\left(g_{1}\right), h_{2}=F\left(g_{2}\right)$ and $h_{3}=f_{3}$. Namely, $\left(h_{1}, h_{2}, h_{3}\right)=F \circ G$. Put

$$
m=p q+p+q, \quad c=(-2)^{p+1} \prod_{i=1}^{p} \frac{i+1}{2 i+1} .
$$

Here is our main result.

Theorem 2.1. Let $p$ and $q$ be natural numbers. Then, $\left(h_{1}, h_{2}, h_{3}\right)$ is a tame automorphism of $k[\mathbf{x}]$ for $n=3$ such that

$$
\begin{gathered}
\operatorname{deg} h_{1}=2 m, \quad \operatorname{deg} h_{2}=(2 p+1) m, \quad \operatorname{deg} h_{3}=m, \\
\operatorname{deg}\left(c^{2} h_{1}^{2 p+1}+h_{2}^{2}\right)=2 p m+p+1 .
\end{gathered}
$$


Note that $\left(h_{1}, h_{2}, h_{3}\right)$ satisfies the assumptions of Lemma 2.1 for $s=2 p+1$. Actually, $(s-1) / 2=p$ and

$$
p \operatorname{deg} h_{1}<2 p m+p+1=(2 p+1) m-(p+1) q+1<\operatorname{deg} h_{2} .
$$

Therefore, we obtain the following corollary to Theorem 2.1.

Corollary 2.1. $\quad$ There exists a tame automorphism $\left(h_{1}^{\prime}, h_{2}^{\prime}, h_{3}^{\prime}\right)$ of $k[\mathbf{x}]$ admitting a reduction of type I such that

$$
\operatorname{deg} h_{1}^{\prime}=2 m, \quad \operatorname{deg} h_{2}^{\prime}=(2 p+1) m, \quad \operatorname{deg} h_{3}^{\prime}=2 p m+p+1
$$

for each $p, q \in \mathbf{N}$, where $m=p q+p+q$.

For a triangular derivation $\Delta$, it is known that the exponential map exp $\Delta$ : $k[\mathbf{x}] \rightarrow k[\mathbf{x}]$ is a tame automorphism of $k[\mathbf{x}]$. If furthermore $\Delta\left(x_{n}\right)=1$, then ker $\Delta=k\left[g_{1}^{\prime}, \ldots, g_{n-1}^{\prime}\right]$, and $\left(g_{1}^{\prime}, \ldots, g_{n-1}^{\prime}, x_{n}\right)$ is a tame automorphism of $k[\mathbf{x}]$. Here, we define

$$
(\exp \Delta)(f)=\sum_{l=0}^{\infty} \frac{\Delta^{l}(f)}{l !}, \quad g_{i}^{\prime}=\sum_{l=0}^{\infty} \frac{\Delta^{l}\left(x_{i}\right)}{l !}\left(-x_{n}\right)^{l}
$$

for each $f \in k[\mathbf{x}]$ and $i=1, \ldots, n-1$ (cf. [2, Sections 1.3 and 6.1]). Hence, $F$ and $G$ are tame automorphisms of $k[\mathbf{x}]$, and so is $F \circ G=\left(h_{1}, h_{2}, h_{3}\right)$. In addition, $E\left(g_{i}\right)=0$ for $i=1,2$. In Section 3, we will consider the polynomial

$$
I=x_{2} x_{3}-x_{1}^{p+1} .
$$

Since $D(I)=0$, it follows that $F(I)=(\exp D)(I)=I$.

To conclude this section, we give explicit descriptions of $f_{i}$ and $g_{j}$ for $i=1,2,3$ and $j=1,2$. By a straightforward computation, we get

$$
f_{1}=x_{1}+x_{2}^{q+1}, \quad f_{2}=x_{2}, \quad g_{1}=x_{1}-x_{3}^{2} .
$$

We show that

$$
f_{3}=x_{3}+\sum_{i=0}^{p}\left(\begin{array}{c}
p+1 \\
i+1
\end{array}\right) x_{1}^{p-i} x_{2}^{(q+1) i+q}, \quad g_{2}=x_{2}+\sum_{i=0}^{p} c_{i} x_{1}^{p-i} x_{3}^{2 i+1},
$$

where

$$
c_{i}=(-2)^{i+1} \prod_{l=0}^{i} \frac{p-l+1}{2 l+1}
$$


for each $i \geq 0$. For the first equality of (2.6), it suffices to verify that

$$
\frac{D^{i+1}\left(x_{3}\right)}{(p+1) !}=\frac{x_{1}^{p-i} x_{2}^{(q+1) i+q}}{(p-i) !}
$$

for $i=0, \ldots, p$. We prove (2.7) by induction on $i$. The case $i=0$ follows from the definition of $D$. Assume that (2.7) is true if $i=l$ for some $0 \leq l<p$. Then,

$$
\begin{aligned}
& \frac{D^{l+2}\left(x_{3}\right)}{(p+1) !}=D\left(\frac{D^{l+1}\left(x_{3}\right)}{(p+1) !}\right)=D\left(\frac{x_{1}^{p-l} x_{2}^{(q+1) l+q}}{(p-l) !}\right) \\
& =\frac{(p-l) x_{1}^{p-l-1} x_{2}^{(q+1) l+q} D\left(x_{1}\right)}{(p-l) !}=\frac{x_{1}^{p-(l+1)} x_{2}^{(q+1)(l+1)+q}}{(p-(l+1)) !} .
\end{aligned}
$$

Hence, (2.7) holds for $i=l+1$, and thus holds for any $0 \leq i \leq p$. Therefore, we have proved the first equality of (2.6). Next, let $g_{2}^{\prime}$ be the right-hand side of the second equality of (2.6). Then, $g_{2}-g_{2}^{\prime}=x_{3} \psi$ for some $\psi \in k[\mathbf{x}]$. To conclude that $g_{2}=g_{2}^{\prime}$, it suffices to show that $E\left(g_{2}^{\prime}-g_{2}\right)=0$, since $E$ is locally nilpotent and $E\left(x_{3}\right) \neq 0$ by definition. In fact, for a locally nilpotent derivation $\Delta$ of $k[\mathbf{x}]$, the condition $\Delta(\phi \psi)=0$ implies $\psi=0$ for $\phi, \psi \in k[\mathbf{x}]$ with $\Delta(\phi) \neq 0$ (cf. [2, Proposition 1.3.32]). It follows that $E\left(g_{2}\right)=0$ as mentioned. Note that $c_{0}=-2(p+1)$, and $2(p-i) c_{i}=-(2 i+3) c_{i+1}$ for $i=0, \ldots, p$. Hence, we have

$$
\begin{aligned}
E\left(g_{2}^{\prime}\right) & =E\left(x_{2}\right)+\sum_{i=0}^{p} c_{i}\left((2 i+1) x_{1}^{p-i} x_{3}^{2 i} E\left(x_{3}\right)+(p-i) x_{1}^{p-i-1} x_{3}^{2 i+1} E\left(x_{1}\right)\right) \\
& =2(p+1) x_{1}^{p}+\sum_{i=0}^{p}\left((2 i+1) c_{i} x_{1}^{p-i} x_{3}^{2 i}+2(p-i) c_{i} x_{1}^{p-i-1} x_{3}^{2 i+2}\right) \\
& =2(p+1) x_{1}^{p}+\sum_{i=0}^{p}\left((2 i+1) c_{i} x_{1}^{p-i} x_{3}^{2 i}-(2 i+3) c_{i+1} x_{1}^{p-(i+1)} x_{3}^{2(i+1)}\right) \\
& =2(p+1) x_{1}^{p}+\sum_{i=0}^{p}(2 i+1) c_{i} x_{1}^{p-i} x_{3}^{2 i}-\sum_{i=1}^{p+1}(2 i+1) c_{i} x_{1}^{p-i} x_{3}^{2 i}=0 .
\end{aligned}
$$

Thus, $E\left(g_{2}-g_{2}^{\prime}\right)=E\left(g_{2}\right)-E\left(g_{2}^{\prime}\right)=0$. Therefore, $g_{2}$ is expressed as in (2.6).

\section{$\S 3 . \quad$ Proof of the Main Result}

In this section, we prove the four equalities of (2.4).

Let $f=\sum_{\alpha \in \mathbf{Z}^{n}} c_{\alpha} \mathbf{x}^{\alpha}$ be a Laurent polynomial in $x_{1}, \ldots, x_{n}$ over $k$, where $c_{\alpha} \in k$ and $\mathbf{x}^{\alpha}=x_{1}^{\alpha_{1}} \cdots x_{n}^{\alpha_{n}}$ for each $\alpha=\left(\alpha_{1}, \ldots, \alpha_{n}\right)$. Then, we set

$$
|f|=\left\{\alpha \in \mathbf{Z}^{n} \mid c_{\alpha} \neq 0\right\} .
$$


For $\eta \in \mathbf{R}^{n}$, we define $\operatorname{deg}_{\eta} f$ to be the maximum among the inner products $\alpha \cdot \eta$ for $\alpha \in|f|$, and put

$$
f^{\eta}=\sum_{\alpha \in \mathbf{Z}^{n}} c_{\alpha}^{\prime} \mathbf{x}^{\alpha}, \quad \text { where } c_{\alpha}^{\prime}=\left\{\begin{array}{lc}
c_{\alpha} & \text { if } \alpha \cdot \eta=\operatorname{deg}_{\eta} f \\
0 & \text { otherwise. }
\end{array}\right.
$$

Clearly, $\operatorname{deg}_{\eta} f=\operatorname{deg}_{\eta} f^{\eta}$ for each $f \in k[\mathbf{x}]$. We note that $(f+g)^{\eta}$ is equal to one of $f^{\eta}, g^{\eta}$ and $f^{\eta}+g^{\eta}$ for each $f, g \in k[\mathbf{x}]$ with $\left|f^{\eta}\right| \cap\left|g^{\eta}\right|=\emptyset$. For a $k$-derivation $\Delta$ of $k[\mathbf{x}]$, we define a $k$-derivation $\Delta^{\eta}$ of $k[\mathbf{x}]$ by setting

$$
\Delta^{\eta}\left(x_{i}\right)=\left\{\begin{array}{cc}
\left(\Delta\left(x_{i}\right)\right)^{\eta} & \text { if } \operatorname{deg}_{\eta}\left(\Delta\left(x_{i}\right) x_{i}^{-1}\right)=\operatorname{deg}_{\eta} \Delta \\
0 & \text { otherwise }
\end{array}\right.
$$

for each $i$, where $\operatorname{deg}_{\eta} \Delta$ denotes the maximum among $\operatorname{deg}_{\eta}\left(\Delta\left(x_{i}\right) x_{i}^{-1}\right)$ for $i=1, \ldots, n$. Then, we have $\Delta^{\eta}\left(f^{\eta}\right)=0$ for each $f \in \operatorname{ker} \Delta$, for otherwise $0 \neq \Delta^{\eta}\left(f^{\eta}\right)=(\Delta(f))^{\eta}$, a contradiction.

Now, we set $\omega_{i}=\operatorname{deg} f_{i}$ for $i=1,2,3$, and $\omega=\left(\omega_{1}, \omega_{2}, \omega_{3}\right)$. Then,

$$
\omega_{1}=q+1, \quad \omega_{2}=1, \quad \omega_{3}=p q+p+q=m .
$$

For each $\alpha=\left(\alpha_{1}, \alpha_{2}, \alpha_{3}\right)$, we have

$$
\operatorname{deg} F\left(\mathbf{x}^{\alpha}\right)=\operatorname{deg} f_{1}^{\alpha_{1}} f_{2}^{\alpha_{2}} f_{3}^{\alpha_{3}}=\alpha_{1} \omega_{1}+\alpha_{2} \omega_{2}+\alpha_{3} \omega_{3}=\operatorname{deg}_{\omega} \mathbf{x}^{\alpha} .
$$

Hence, $\operatorname{deg} F(f) \leq \operatorname{deg}_{\omega} f$ for each $f \in k[\mathbf{x}]$. The equality holds when $f^{\omega}$ is a term. By (2.5) and (2.6), we see that $g_{1}^{\omega}=-x_{3}^{2}$ and $g_{2}^{\omega}=c_{p} x_{3}^{2 p+1}$ are terms, so $\operatorname{deg} h_{i}=\operatorname{deg} F\left(g_{i}\right)=\operatorname{deg}_{\omega} g_{i}$ for $i=1,2$. Hence, $\operatorname{deg} h_{1}=2 m$ and $\operatorname{deg} h_{2}=(2 p+1) m$. In addition, $\operatorname{deg} h_{3}=\operatorname{deg} f_{3}=m$. Thus, we have proved the first three equalities of (2.4).

Next, we consider the polynomial $P:=c^{2} g_{1}^{2 p+1}+g_{2}^{2}$. Our goal is to establish that $\operatorname{deg} F(P)=2 p m+p+1$, which immediately implies the last equality of (2.4). Write $P=P_{1}+P_{2}$, where $\phi=g_{2}-x_{2}$,

$$
P_{1}=c^{2} g_{1}^{2 p+1}+\phi^{2} \quad \text { and } \quad P_{2}=x_{2}^{2}+2 \phi x_{2} .
$$

Set $\epsilon=(1,0,-2)$. Then, $g_{1}^{2 p+1}$ and $\phi^{2}$ belong to $x_{3}^{2(2 p+1)} k\left[\mathbf{x}^{\epsilon}\right]$, since $g_{1}$ and $\phi$ are in $x_{3}^{2} k\left[\mathbf{x}^{\epsilon}\right]$ and $x_{3}^{2 p+1} k\left[\mathbf{x}^{\epsilon}\right]$ by (2.5) and (2.6), respectively. Hence,

$$
P_{1}^{\omega}=c^{\prime} \mathbf{x}^{u \epsilon} x_{3}^{2(2 p+1)}=c^{\prime} x_{1}^{u} x_{3}^{2(2 p-u+1)}, \text { where } u \geq 0, c^{\prime} \in k \backslash\{0\} .
$$

We claim that $u \neq 0$. In fact, the monomial $x_{3}^{2(2 p+1)}$ appears in $g_{1}^{2 p+1}$ and $\phi^{2}$ with coefficients -1 and $c_{p}^{2}$, respectively. By definition, $c_{p}=c$. Hence, 
$x_{3}^{2(2 p+1)}$ does not appear in $P_{1}$, so we get $u \neq 0$. On the other hand, $P_{2}^{\omega}=$ $x_{2}\left(x_{2}+2 \phi\right)^{\omega}=2 x_{2} \phi^{\omega}=2 c x_{2} x_{3}^{2 p+1}$. Clearly, $\left|P_{1}^{\omega}\right| \cap\left|P_{2}^{\omega}\right|=\emptyset$. Hence, $P^{\omega}$ must be equal to $P_{1}^{\omega}$ or $P_{2}^{\omega}$ or $P_{1}^{\omega}+P_{2}^{\omega}$. Recall that $E\left(g_{i}\right)=0$ for $i=1,2$. So, $E(P)=0$. This implies that $E^{\omega}\left(P^{\omega}\right)=0$ as mentioned. A straightforward computation shows that

$$
\begin{aligned}
& \operatorname{deg}_{\omega}\left(E\left(x_{1}\right) x_{1}^{-1}\right)=\operatorname{deg}_{\omega} x_{1}^{-1} x_{3}=-(q+1)+m=p q+p-1, \\
& \operatorname{deg}_{\omega}\left(E\left(x_{2}\right) x_{2}^{-1}\right)=\operatorname{deg}_{\omega} x_{1}^{p} x_{2}^{-1}=p(q+1)-1=p q+p-1, \\
& \operatorname{deg}_{\omega}\left(E\left(x_{3}\right) x_{3}^{-1}\right)=\operatorname{deg}_{\omega} x_{3}^{-1}=-m=-p q-p-q<p q+p-1 .
\end{aligned}
$$

Accordingly, we get $E^{\omega}\left(x_{i}\right)=\left(E\left(x_{i}\right)\right)^{\omega}=E\left(x_{i}\right)$ for $i=1,2$ and $E^{\omega}\left(x_{3}\right)=0$. Then, it follows that $E^{\omega}\left(P_{i}^{\omega}\right) \neq 0$ for $i=1,2$. Consequently,

$$
\begin{gathered}
P^{\omega}=P_{1}^{\omega}+P_{2}^{\omega}=c^{\prime} x_{1}^{u} x_{3}^{2(2 p-u+1)}+2 c x_{2} x_{3}^{2 p+1}, \\
0=E^{\omega}\left(P^{\omega}\right)=2 u c^{\prime} x_{1}^{u-1} x_{3}^{4 p-2 u+3}+4 c(p+1) x_{1}^{p} x_{3}^{2 p+1},
\end{gathered}
$$

so $u=p+1$ and $c^{\prime}=-2 c$. Therefore, we get

$$
P^{\omega}=-2 c x_{1}^{p+1} x_{3}^{2 p}+2 c x_{2} x_{3}^{2 p+1}=2 c x_{3}^{2 p}\left(x_{2} x_{3}-x_{1}^{p+1}\right)=2 c x_{3}^{2 p} I .
$$

Hence, $\operatorname{deg}_{\omega} P=2 p m+m+1$. Since $F(I)=I$ as mentioned,

$$
\operatorname{deg} F\left(P^{\omega}\right)=\operatorname{deg}\left(2 c f_{3}^{2 p} I\right)=2 p m+p+1 .
$$

Finally, let $Q=P-P^{\omega}$. Since $F(P)=F\left(P^{\omega}\right)+F(Q)$, it remains only to verify that $\operatorname{deg} F(Q)<2 p m+p+1$ by (3.1). Observe that $P$ and $Q$ belong to $x_{2}^{2} k\left[x_{2}^{-1} x_{3}^{2 p+1}, \mathbf{x}^{\epsilon}\right]$. Furthermore, $\operatorname{deg}_{\omega} \mathbf{x}^{\epsilon}=q+1-2 m<0$, and

$$
\operatorname{deg}_{\omega} x_{2}^{-1} x_{3}^{2 p+1}=(2 p+1) m-1=-(p+1) \operatorname{deg}_{\omega} \mathbf{x}^{\epsilon} .
$$

Hence, $\operatorname{deg}_{\omega} Q \equiv \operatorname{deg}_{\omega} P\left(\bmod \operatorname{deg}_{\omega} \mathbf{x}^{\epsilon}\right)$. Since $\operatorname{deg}_{\omega} Q<\operatorname{deg}_{\omega} P$, we get

$$
\begin{aligned}
& \operatorname{deg}_{\omega} Q \leq \operatorname{deg}_{\omega} P+\operatorname{deg}_{\omega} \mathbf{x}^{\epsilon}=2 p m+m+1+q+1-2 m \\
& \quad=2 p m+p+1-p(q+2)+1<2 p m+p+1 .
\end{aligned}
$$

Thus, $\operatorname{deg} F(Q) \leq \operatorname{deg}_{\omega} Q<2 p m+p+1$, and thereby proving the last equality of $(2.4)$.

\section{§4. Remarks}

As far as we know, the answer to the following simple question is not known. 
Question 4.1. Do there exist polynomials $f, g \in k[\mathbf{x}]$ for $n=3$ as follows?

(i) $k[f, g, h]=k[\mathbf{x}]$ for some $h \in k[\mathbf{x}]$.

(ii) $\operatorname{deg}\left(f^{3}+g^{2}\right) \leq \operatorname{deg} f$.

This question is closely related to the study of $\mathrm{Aut}_{k} k[\mathbf{x}]$ for $n=3$. In fact, no automorphism of $k[\mathbf{x}]$ admits a reduction of type II or III or IV if the answer to Question 4.1 is negative. The reason is as follows.

Suppose that there exists an automorphism of $k[\mathbf{x}]$ admitting a reduction of type II or III or IV. Then, it follows from [10, Definitions 2, 3 and 4] that there exists an automorphism $\left(g_{1}, g_{2}, g_{3}\right)$ as follows:

(1) $\operatorname{deg} g_{1}=2 l$ and $\operatorname{deg} g_{2}=3 l$ for some $l \in \mathbf{N}$.

(2) There exists $\phi \in k\left[g_{1}, g_{2}\right] \backslash k$ with $\operatorname{deg} \phi \leq 2 l$ such that $\bar{\phi}$ and $\bar{g}_{1}$ are linearly independent over $k$.

Since $\operatorname{deg} \phi \leq \operatorname{deg} g_{1}$ and $\operatorname{deg} \phi<\operatorname{deg} g_{2}$, the condition (2) implies that $\bar{\phi} \notin k\left[\bar{g}_{1}, \bar{g}_{2}\right]$. Write $\phi=\sum_{i, j} c_{i, j} g_{1}^{i} g_{2}^{j}$, where $c_{i, j} \in k$ for each $i$ and $j$. Let $u_{1}$ and $u_{2}$ be the maximal numbers such that $c_{u_{1}, j^{\prime}} \neq 0$ and $c_{i^{\prime}, u_{2}} \neq 0$ for some $j^{\prime}$ and $i^{\prime}$, and let $q_{i}$ and $r_{i}$ respectively be the quotient and residue of $u_{i}$ divided by $e_{i}$ for $i=1,2$, where

$$
e_{1}=\frac{\operatorname{deg} g_{2}}{\operatorname{gcd}\left(\operatorname{deg} g_{1}, \operatorname{deg} g_{2}\right)}=3, \quad e_{2}=\frac{\operatorname{deg} g_{1}}{\operatorname{gcd}\left(\operatorname{deg} g_{1}, \operatorname{deg} g_{2}\right)}=2 .
$$

Then, due to [9, Theorem 3] (see also [5], [7] and [11]), it follows that

$$
\begin{aligned}
\operatorname{deg} \phi & \geq q_{i}\left(\operatorname{lcm}\left(\operatorname{deg} g_{1}, \operatorname{deg} g_{2}\right)-\operatorname{deg} g_{1}-\operatorname{deg} g_{2}+\operatorname{deg}\left[g_{1}, g_{2}\right]\right)+r_{i} \operatorname{deg} g_{i} \\
& \geq q_{i}(l+2)+r_{i} \operatorname{deg} g_{i}
\end{aligned}
$$

for $i=1,2$. Since $\operatorname{deg} g_{1}=2 l$ and $\operatorname{deg} \phi \leq 2 l$ by assumption, $\left(q_{1}, r_{1}\right)$ must be $(0,1)$ or $(1,0)$. Hence, $u_{1}=3 q_{1}+r_{1}$ is equal to 1 or 3 . Similarly, $\left(q_{2}, r_{2}\right)=(1,0)$, and so $u_{2}=2$. In particular, $u_{1} \leq 3$ and $u_{2}=2$. The polynomials $g_{1}^{i} g_{2}^{j}$ for $i=0,1,2,3$ and $j=0,1,2$ with $(i, j) \neq(3,0),(0,2)$ have distinct total degrees. This implies that $c_{i, j}=0$ for each $(i, j)$ with $2 i+3 j>6$, while $c_{3,0} \neq 0$ and $c_{0,2} \neq 0$, for otherwise $\bar{\phi}=c_{i, j} \bar{g}_{1}^{i} \bar{g}_{2}^{j}$ for some $(i, j)$, which contradicts that $\bar{\phi} \notin k\left[\bar{g}_{1}, \bar{g}_{2}\right]$. Hence,

$$
\phi=c_{3,0} g_{1}^{3}+c_{0,2} g_{2}^{2}+c_{1,1} g_{1} g_{2}+c_{2,0} g_{1}^{2}+c_{0,1} g_{2}+c_{1,0} g_{1}+c_{0,0},
$$

in which $c_{3,0} \neq 0$ and $c_{0,2} \neq 0$. Without loss of generality, we may assume that $c_{0,2}=1$. Then, (4.1) is expressed as

$$
\phi=c_{3,0} \hat{f}^{3}+\hat{g}^{2}+b \hat{f}+c, \text { where } \hat{f}=g_{1}+a, \hat{g}=g_{2}+\frac{c_{1,1}}{2} g_{1}+\frac{c_{0,1}}{2}
$$


and $a, b, c \in k$. Indeed, $\phi=c_{3,0} g_{1}^{3}+\hat{g}^{2}+c_{2,0}^{\prime} g_{1}^{2}+c_{1,0}^{\prime} g_{1}+c_{0,0}^{\prime}$ for some $c_{2,0}^{\prime}, c_{1,0}^{\prime}, c_{0,0}^{\prime} \in k$. Then, we have (4.2) for $a=c_{0,2}^{\prime} /\left(3 c_{3,0}\right)$ and some $b, c \in k$. Finally, put $f=c_{3,0} \hat{f}$ and $g=c_{3,0} \hat{g}$. Clearly, $\operatorname{deg} f=\operatorname{deg} g_{1}=2 l, \operatorname{deg} g=$ $\operatorname{deg} g_{2}=3 l$, and $k\left[f, g, g_{3}\right]=k[\mathbf{x}]$. Moreover,

$$
\operatorname{deg}\left(f^{3}+g^{2}\right)=\operatorname{deg} c_{3,0}^{2}\left(c_{3,0} \hat{f}^{3}+\hat{g}^{2}\right) \leq 2 l=\operatorname{deg} f
$$

by (4.2), since the total degrees of $\phi$ and $b \hat{f}+c$ are at most $2 l$. Therefore, $f$ and $g$ satisfy the conditions of Question 4.1.

It is worthwhile to mention that, if there exists a tame automorphism $\left(h_{1}, h_{2}, h_{3}\right)$ with $\operatorname{deg} h_{1}: \operatorname{deg} h_{2}: \operatorname{deg} h_{3}=2: 3: 1$ and $\operatorname{deg}\left(c h_{1}^{3}+h_{2}^{2}\right) \leq \operatorname{deg} h_{1}$ for some $c \in k \backslash\{0\}$, then we can construct a tame automorphism admitting a reduction of type II or III. On the other hand, if $p=1$, then (2.4) gives that $\operatorname{deg} h_{1}=2 m, \operatorname{deg} h_{2}=3 m, \operatorname{deg} h_{3}=m$ and $\operatorname{deg}\left(c^{2} h_{1}^{3}+h_{2}^{2}\right)=2 m+2$. In this case, we have $m=2 q+1$ and

$$
\frac{\operatorname{deg} h_{1}}{\operatorname{deg}\left(c^{2} h_{1}^{3}+h_{2}^{2}\right)}=\frac{2 m}{2 m+2} \rightarrow 1 \quad(q \rightarrow \infty),
$$

although $\operatorname{deg}\left(c^{2} h_{1}^{3}+h_{2}^{2}\right)>\operatorname{deg} h_{1}$.

Assume that $f, g \in k[\mathbf{x}]$ are algebraically independent over $k$ for which $\operatorname{deg} f: \operatorname{deg} g=r: s$, where $r, s \in \mathbf{N}$ with $2 \leq r<s$ and $\operatorname{gcd}(r, s)=1$. Then, it easily follows from [9, Theorem 3] (see also [5, Theorem 2.1]) that

$$
\operatorname{deg}\left(f^{s}+g^{r}\right)> \begin{cases}\operatorname{deg} g & \text { if } r \geq 3 \\ \operatorname{deg} f & \text { if } r=2 \text { and } s \geq 5 .\end{cases}
$$

Hence, $\operatorname{deg}\left(f^{s}+g^{r}\right) \leq \operatorname{deg} f$ is possible only if $(r, s)=(2,3)$. We define $f, g \in k\left[x_{1}, x_{2}\right]$ by

$$
f=-x_{1}^{4 l} x_{2}^{2(2 m-1)}-2 x_{1}^{l} x_{2}^{m}, \quad g=x_{1}^{6 l} x_{2}^{3(2 m-1)}+3 x_{1}^{3 l} x_{2}^{3 m-1}+\frac{3}{2} x_{2},
$$

where $l, m \in \mathbf{N}$. Then, $\operatorname{deg} f: \operatorname{deg} g=2: 3$. Moreover, $f$ and $g$ are algebraically independent over $k$, and

$$
f^{3}+g^{2}=x_{1}^{3 l} x_{2}^{3 m}+\frac{9}{4} x_{2}^{2},
$$

since $f=-x_{1}^{l} x_{2}^{m}\left(\mathbf{x}^{\alpha}+2\right)$ and $g=x_{2}\left(\mathbf{x}^{2 \alpha}+3 \mathbf{x}^{\alpha}+3 / 2\right)$, where $\alpha=(3 l, 3 m-2)$. In particular, $\operatorname{deg}\left(f^{3}+g^{2}\right)=\operatorname{deg} f$ if $l=m=1$, and $\operatorname{deg}\left(f^{3}+g^{2}\right)<\operatorname{deg} f$ otherwise. If $k$ is of characteristic $r>0$, then $f=x_{1}^{r l}$ and $g=x_{2}+x_{1}^{s l}$ satisfy $f^{s}-g^{r}=-x_{2}^{r}$ for any $l, s \in \mathbf{N}$. Hence, $\operatorname{deg}\left(f^{s}-g^{r}\right) \leq \operatorname{deg} f$ in this case. 
Note: Instead of Question 4.1, the author first asked a question whether there exist $f, g \in k[\mathbf{x}]$ with $\operatorname{deg}\left(f^{3}+g^{2}\right) \leq \operatorname{deg} f$ which are algebraically independent over $k$. In answer to the question, Prof. Hiraku Kawanoue informed him of an example satisfying $\operatorname{deg}\left(f^{3}+g^{2}\right)=\operatorname{deg} f$. The example (4.3) is a modification of Kawanoue's example by the author.

Recently, the author [6] showed that no tame automorphism of $k[\mathbf{x}]$ for $n=3$ admits a reduction of type IV.

\section{References}

[1] A. van den Essen, L. Makar-Limanov and R. Willems, Remarks on Shestakov-Umirbaev, Report 0414, Radboud University of Nijmegen, Toernooiveld, 6525 ED Nijmegen, The Netherlands, 2004.

[2] A. van den Essen, Polynomial automorphisms and the Jacobian conjecture, Progr. Math., 190, Birkhäuser, Basel, 2000.

[3] H. W. E. Jung, Über ganze birationale Transformationen der Ebene, J. Reine Angew. Math. 184 (1942), 161-174.

[4] W. van der Kulk, On polynomial rings in two variables, Nieuw Arch. Wiskunde (3) 1 (1953), 33-41.

[5] S. Kuroda, A generalization of the Shestakov-Umirbaev inequality, J. Math. Soc. Japan 60 (2008), no. 2, 495-510.

[6] _ Shestakov-Umirbaev reductions and Nagata's conjecture on a polynomial automorphism, arXiv:math.AC/0801.0117.

[7] L. Makar-Limanov and J.-T. Yu, Degree estimate for subalgebras generated by two elements, J. Eur. Math. Soc. (JEMS) 10 (2008), no. 2, 533-541.

[8] M. Nagata, On automorphism group of $k[x, y]$, Department of Mathematics, Kyoto University, Lectures in Mathematics, No. 5, Kinokuniya Book Store, Tokyo, 1972.

[9] I. P. Shestakov and U. U. Umirbaev, Poisson brackets and two-generated subalgebras of rings of polynomials, J. Amer. Math. Soc. 17 (2004), no. 1, 181-196 (electronic).

[10] J. Amer. Math. Soc. 17 (2004), no. 1, 197-227 (electronic).

[11] S. Vénéreau, A parachute for the degree of a polynomial in algebraically independent ones, arXiv:math.AC/0704.1561. 